\title{
Effectiveness of planned teaching programme on practice of post natal exercises among mothers who have undergone lower segment cesarean section
}

\section{Vijaya Rani M. ${ }^{1}$, Maryam Ghanbari Andarieh ${ }^{2 *}$, Fatemeh Hoseinpoor Heidary ${ }^{2}$}

\author{
${ }^{1}$ Rajiv Gandhi University of Health Sciences, Karnataka, Bangalore, India \\ ${ }^{2}$ Infertility and Reproductive Health Research Center, Health research Institute, Babol University of Medical Sciences, \\ Babol, Iran
}

Received: 24 August 2016

Accepted: 24 September 2016

*Correspondence:

Dr. Maryam Ghanbari Andarieh,

E-mail: goolemaryam49@yahoo.com

Copyright: (C) the author(s), publisher and licensee Medip Academy. This is an open-access article distributed under the terms of the Creative Commons Attribution Non-Commercial License, which permits unrestricted non-commercial use, distribution, and reproduction in any medium, provided the original work is properly cited.

\section{ABSTRACT}

Background: To assess the effectiveness of planned teaching programme on practice of post natal exercises among mothers who have undergone Lower segment cesarean section (L.S.C.S) in selected hospital at Bangalore.

Methods: compare the practice scores of postnatal exercises among LSCS mothers between control group and experimental group.

Results: Results shows that in post test the control group had inadequate practice score with a mean of $16.33 \%$, standard deviation of 9.44 and mean percentage of $19.60 \%$ where as in post test the experimental group there was a significant mean practice score gain of $39.84 \%$ and standard deviation of 12.58 and mean percentage $79.67 \%$ with a differences of $60.07 \%$.

Conclusions: Significant difference was found between control and experimental group practice score of mothers in all areas of post natal exercises.

Keywords: Benefit of postnatal exercise, Complication, Incidence, Postnatal exercise, LSCS, Postnatal depression

\section{INTRODUCTION}

With the current National Policy for health promotion there is an emphasis on increasing the understanding of the population about the value of different forms of exercise and health. It is difficult to imagine that a woman with a new baby will not undertake quiet a substantial amount of exercise once she has recovered from the birth event, but women vary considerably in their perception of exercise. ${ }^{1}$

The pregnancy is a state in which women of child bearing age are in a period of growth and development of fetus in womb. Pregnancy is rare below 12 years and beyond 50 years. $^{2}$ By early twentieth century the "classical" operation has become quite widespread for obstructed labor and placenta previa when Man ro-kerr introduced the concepts of LSCS in 1920's the profession. Nevertheless persisted the new procedure which has now become the standard intervention for complicated labor world wise. The rate of caesarean sections is increasing and this may be attributed to both technological and social changes. ${ }^{3}$ Stress incontinence occurs in some women following delivery. Pelvic floor exercises will help to rehabilitate the pelvic floor but the women will usually require specific obstetric exercises prescribed by her physician. ${ }^{4}$ It's vital not to rush back into exercises after caesarean section. Routine should be gentle and should avoid major abdominal work for the first three months are should be taken when exercising following a 
caesarean. Basic exercise is exercises that involve a large number of muscles in the body. They are generally multijoint movements that target the larger muscles of the body such as chest, back and thighs but also involve the smaller muscles as well as the shoulders, arms, calves, abdominal and auxiliary muscles. During pregnancy, labor and the puerperium mother should be influenced to incorporate a sensible approach to exercises. Relaxation, walking and other form of exercises can be encouraged as part of general lifestyle. Specific exercises for strengthening pelvic floor and abdominal muscles or relieving aches and pain such as cramps or backache will be useful even beyond the month of childbearing. ${ }^{5}$

Caesarean section is a life-saving procedure firmly ensconced in obstetric practice. With the advances in anesthetics services and improved surgical techniques, the morbidity and mortality of this procedure have come down considerably. Over the last 20 years there has been a disturbing increase in the rate of Caesarean sections in India. ${ }^{6}$ A regular pattern of exercises becomes an ingrained habit; however interruptions such as lifestyle transitions can distract the most intentioned and most important exercises. There has been a gradual acceptance that exercises are beneficial for women during any part of the reproductive process. ${ }^{7}$ Postpartum recovery period is usually longer for cesarean births physiological concern of women after cesarean birth may include fatigue activity intolerance and incision problems .Nearly $11.5 \%$ of post natal mothers die with puerperal sepsis in India and also found that $64 \%$ of mothers are suffering from back pain and other puerperal complications because of not doing exercise. The number of women delivering by $\mathrm{CS}$ is increasing. ${ }^{8}$ A study was conducted to estimate the maternal Morbidity associated with cesarean deliveries when compared with spontaneous vaginal delivery. The study result showed that of $(\mathrm{P}=0.3) \mathrm{Vs}(\mathrm{P}=.01)$.In India the maternal mortality and morbidity from L.S.C.S is 4-6 times greater than vaginal delivery. ${ }^{10}$

During the clinical experiences, the investigators found that post-natal exercises were neglected by mothers due to lack of practice and many mothers home developed Post-operative complication. ${ }^{17}$

A study was conducted on incidence of cesarean birth. The study result showed in Brazil (36\%) UK (21.4\%), USA (24.4\%), Italy (22.4\%), Sweden (11.9\%) Taiwan $(32.34 \%)$, Netherland $(9.5 \%)$ studies have stated that the official statistics the rate of L.S.C.S not more than $15 \%$ with the primary L.S.C.S being no more than $12 \%$ of total births. $^{18}$

A study was conducted on the incidence of cesarean birth at KEM hospital; name none from $1-95 \%$ to $5.5 \%$ in. Maternal Mortality rate was 6.1/ 1, 00,000 and who also stated that major sources of morbidity and associated mortality related to complications are maternal sepsis and thrombotic deceases. The author concluded that L.S.C.S Technique appears to be a safe and efficient method and associated with minimal post operative infections morbidity. ${ }^{19}$

A study was conducted on determinants of caesarean section rates in India delivery complication, They examines the levels and correlates of delivery related complications and caesarean section deliveries in 18th selected states of India in terms of specific maternal and institutional factors using data from the national family health survey. The study result showed that in 199293.goa $15.3 \%$ and Kerala $13.7 \%$ were the two states with relatively higher caesarean section rates. ${ }^{20} \mathrm{~A}$ study was conducted on Elective babies born before 39th week. The study result showed that face risks more than 24,000 women who had repeat $\mathrm{C}$-sections performed at 37 weeks or later at 19 medical centers in the United States between 1999 and 2002. Of those, 13,258 women had elective C-sections. ${ }^{21}$ A study was conducted on benefits of a structured exercise and socialization program in combating postnatal depression and related conditions. The study concluded that PND can lead to on going health and social problems for the mother and her family. It has been suggested that group exercise activities such as pram-walking by their nature encompass both exercise and social support aspects, hence address both biological and psychosocial components of PND. ${ }^{22}$ A study was conducted on "effect of the pelvic floor exercise on post partum symptoms of stress urinary incontinence and pelvic muscle strength in prim gravid during post partum." The study concluded that practice of pelvic exercise by primipara resulted in fewer urinary incontinence symptoms during late post partum $(\mathrm{P}=$ 0.32). The pelvic floor muscles offer support for the bowel, bladder and uterus. After having a baby, the pelvic floor can become weakened, leading to urinary and bowel incontinence. Fortunately, there are several exercises that can help tighten up the pelvic floor muscles, lessening the chance of any embarrassing accidents. ${ }^{24}$ A study was conducted on teaching and practice of pelvic floor muscle exercise in Primipara's women during pregnancy and the postpartum period. Primipara's women with term singleton delivery were interviewed 6 months after delivery.

The study concluded that tremendous potential for the improvement of pelvic floor muscle exercise education and targeting at risk women in ante partum period. ${ }^{25}$ An article reported on the nurse needs to educate each mother individually and to plan health teaching accordingly that kind of institution or teaching is focused on various aspects. Post natal exercises, care and neonatal care will save the mother in many anxious movements when she is at hospital. The nurse also can ease the transition from pregnancy to motherhood. Therefore, it is the nurses responsibility to provide the women and their gamily with adequate knowledge on healthier information to bring the gap between the knowledge and health practice to the mother. ${ }^{26}$ 


\section{METHODS}

The conceptual framework for the study was derived from general system theory.

The independent variable is the planned teaching programme regarding post natal exercises. The dependent variable is practice of LSCS mothers regarding post natal exercises. A total number of 50 mothers who met in the inclusion criteria were selected by using simple random sampling technique by lottery method.

To meet the objectives of the study the tool was developed by the investigator. The tool used for the study comprised of an observational checklist and a planned teaching programme on practice of postnatal exercise among LSCS mothers.

Observational checklist consists of two parts.

Part I: It deals with demographic variables which include age, educational status, occupation, religion, type of family, previous knowledge, and sources of information.

Part II: It consists of 50 practice questions regarding post natal exercises for L.S.C.S. mothers with maximum obtainable score.

A planned teaching programme was also prepared by the investigator which covers major areas such as introduction, meaning, definition, benefits, exercises following caesarean section, care of incision area while practicing exercises and complication of negligence of exercises.
The existing practice of the samples was assessed using an observational checklist.

A team of 7 experts ensured content validity of the tool. The content validity of the tool and the Teaching Programme were established in consultation with guide and experts from the field of obstetrics, Maternity nursing, and the tools were modified according to the suggestions and recommendations of the experts.

Reliability of the tool and teaching programme were tested by implementing the tool on 10 mothers who had undergone LSCS, who fulfilled the inclusion criteria in other than the sample area. These samples were excluded from the main study. Reliability was established by test/ retest method. 10 samples were used to check the reliability through test re test method. The interval between the two tests was 8 days. The correlation co efficient was calculated using Karl Pearson method, $r=$ 0.8 , was high positive. The observational checklist was found to be reliable.

\section{RESULTS}

Table 1 shows that about distribution of control and experimental group mothers according to their age group depicts that the highest percentage $40 \%$ and $36 \%$ respectively of mothers from both the group were in the age of 21-25 years and $36 \%$ of mothers in control were in the age group of 25-30 years. The lowest percentage only $8 \%$ of mothers from the control group was in the age group of 31-35 years and $28 \%$ of mothers from the experimental group were in the age group of 26-30 years. It might be associated with normal reproductive age in India.

Table 1: Area-wise comparison of mean, SD and mean percentage of control and experimental group practices scores regarding post natal exercise.

\begin{tabular}{|lllllllll|}
\hline Area wise & Area score & \multicolumn{3}{c}{ Control group } & \multicolumn{4}{c|}{ Experimental group } \\
& & Mean & SD & Mean \% & Mean & SD & Mean \% & \\
\hline Circulatory & 06 & 1.72 & 1.68 & 28 & 4.72 & 1.31 & 78.66 & 50.66 \\
\hline Abdominal breathing & 10 & 3.62 & 1.80 & 18 & 8.08 & 2.99 & 80.80 & 62.8 \\
\hline Abdominal tightening & 06 & 1.89 & 1.32 & 22 & 4.88 & 1.79 & 81.33 & 59.33 \\
\hline Pelvic tilting & 10 & 3.27 & 1.72 & 17.2 & 8.04 & 2.90 & 80.4 & 63.2 \\
\hline Knee rolling & 09 & 2.93 & 1.48 & 16.44 & 6.68 & 0.95 & 74.22 & 57.78 \\
\hline Hip hitching & 09 & 2.93 & 1.44 & 16 & 7.44 & 2.64 & 82.66 & 66.66 \\
\hline Over all & 50 & 16.33 & 9.44 & 19.60 & 39.84 & 12.58 & 79.67 & 60.07 \\
\hline
\end{tabular}

Distribution of control and experimental group mothers according to their religion reveals that the highest percentage $52 \%$ and $40 \%$ from control and experimental group were Hindu .Similarly $36 \%$ of mothers from control and experimental group were Muslim and Christian. The Lowest $12 \%$ of mothers from the control group were Christians. It reveals that more or less similarly all mothers from both groups were Hindu.

Distribution of control and experimental group mothers according to their types of family reveals that the highest percentage $60 \%$ and $72 \%$ respectively of mothers from 
nuclear family. Similarly $40 \%$ and $28 \%$ of mothers from the control and experimental group were joint family. It seems that majority of mothers from the both groups were nuclear family.

Distribution of control and experimental group mothers according to their source of information reveals that the higher percentage $44 \%$ respectively of mothers from both had received from the mass media. $28 \%$ of mothers from the control group had received information from the health workers. The lowest $12 \%$ of mother from the experimental group and had received information from neighbours. It reveals that the mothers had received information more from the mass media.

Area wise Comparison of mean, SD, mean percentage scores showed that control group the highest mean percentage on circulatory exercise were (1.68 \pm 1.72$)$ which is $28 \%$. The lowest mean percentage on hip hitching $(1.44 \pm 2.90)$ which is $16 \%$ of the maximum scores with a different of $12 \%$.

Further for the experimental group the highest mean percentage on hip hitching exercise were $(7.44 \pm 2.64)$ which is $82.66 \%$ of the maximum scores. The lowest mean percentage on knee rolling $(6.68 \pm 0.95)$ which is $74.22 \%$ scores with a different of $8.44 \%$. Similarly the overall mean percentage for the control group was $19.60 \%$ where after the experimental group overall one $79.67 \%$ with a differences of $60.07 \%$ it seems that the teaching programme regarding postnatal exercises overall were effective.

Comparison of practice score of control and experimental group regarding circulatory exercise shows that $52 \%$ of mothers from the control group had sit comfortably on bed. $32 \%$ mothers had supported her legs on either by hands or on bed and $20 \%$ mothers stretched the ankle 12 times at a time. $24 \%$ mothers rotated the feet at the circle 12 times at a time and hold the knees and count for four. The lowest $16 \%$ of mothers repeated all steps for 10 times.

However in experimental group all mothers (100\%) had sit comfortably on bed and more less similarly $80 \%$ and $76 \%$ of mothers bent and stretched the ankles 12 times at one time and $68 \%$ mothers rotated the feet at the circular rotation 12 times at one time, $72 \%$ of hold the knees and count for four and $76 \%$ of mothers repeated all steps 10 times at a time.

Further comparison of difference between control and experimental group shows that the highest $60 \%$ of mothers repeated all steps 10 times. The lowest $44 \%$ of mother rotates the feet at circle the feet at the ankle at least 12 times reveals that effectiveness of teaching programme on circular exercise (Figure 1).

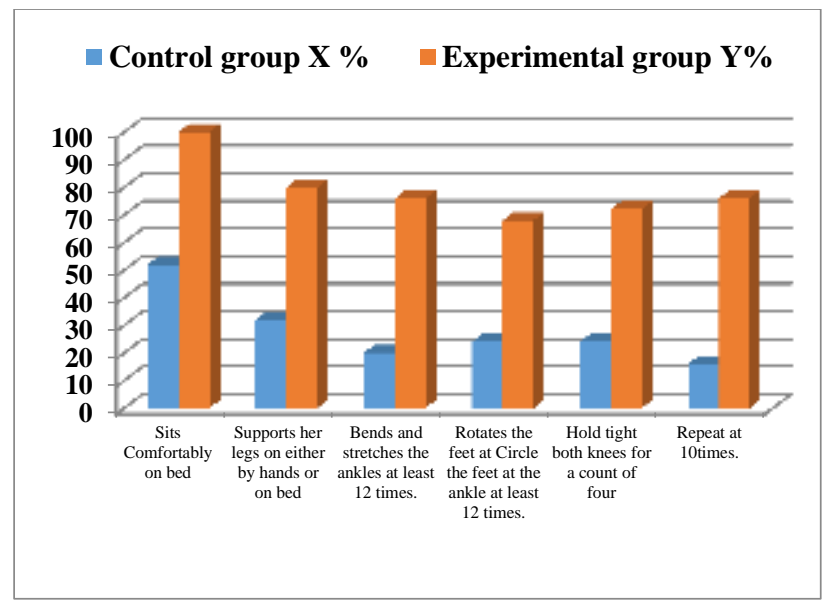

Figure 1: Comparison of control and experimental group correct practice scores regarding circulatory exercise on observation.

Comparison of practice scores of control and experimental group regarding Abdominal breathing exercise shows that the highest percentage $44 \%$ of mothers from the control group had sit comfortably on bed and $36 \%$ of mother eyes should be closed. $28 \%$ of mothers listened the breathing and $20 \%$ of mothers raising her abdominal wall, Exhale slowly and Place one hand on the chest and on the abdomen. The lowest percentage $12 \%$ of mothers repeated all steps for 5 times.

However in experimental group, all mothers (100) had sit comfortably on bed. similarly $96 \%$ and $88 \%$ of mothers placed one hands on the chest and the abdomen and eyes should be closed.80\% of mothers raised her abdominal wall, exhale slowly and $76 \%$ of mothers listening the breathing especially out ward breathing and repeated all steps for 5 times. $72 \%$ of mothers the hand and the chest should remain stationary and the lowest percentage 6 $4 \% \%$ of mothers when inhaling, the hand on the abdomen should be raised.

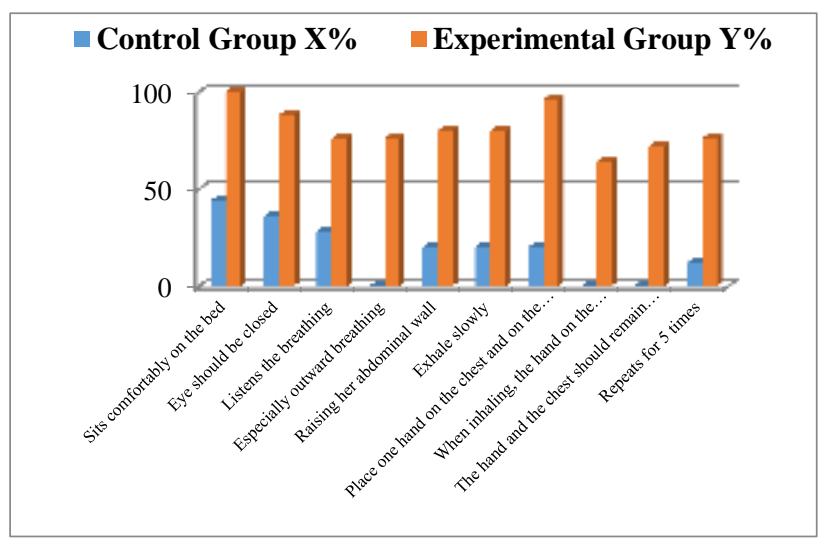

Figure 2: Items-wise comparison of control and experimental group correct practice scores regarding abdominal breathing exercise on observation. 
Further comparison of difference between control and experimental group, $76 \%$ of mothers listened the breathing especially inward and outward breathing, placed one hands on the chest and the abdomen and the lowest $48 \%$ of mothers had listens the breathing (Figure 2) reveals that the effectiveness of breathing programme on abdominal breathing exercise.

Comparison of practice scores of control and experimental group regarding abdominal tightening exercise regarding the highest percentage $40 \%$ of mothers Sits comfortably on bed the higher $36 \%$ of mothers relaxed the body slowly. $24 \%$ of mothers hold the abdomen for 10 second. The lowest percentages $16 \%$ of mothers pulled the lower part of the abdomen in and repeat for 12 times at a time.

However in experimental group, all mothers $100 \%$ had sit comfortably on bed. The highest $92 \%$ of mothers relaxed the body slowly. Similarly $84 \%$ and $80 \%$ of mothers and repeated all steps at a time. Similarly $72 \%$ of mothers had taken deep breathing in and out. The lowest percentage $60 \%$ of mothers pulled the lower part of abdomen in.

Further comparison of difference between control and experimental group, reveals that the highest $68 \%$ of mothers repeated all steps at a time. The lowest $56 \%$ of mothers had taken deep breathing in and out, hold the abdomen for $10 \mathrm{Sec}$ and relaxed the body slowly reveals that the effectiveness of Teaching Programme on (Figure 3) abdominal tighten exercise.

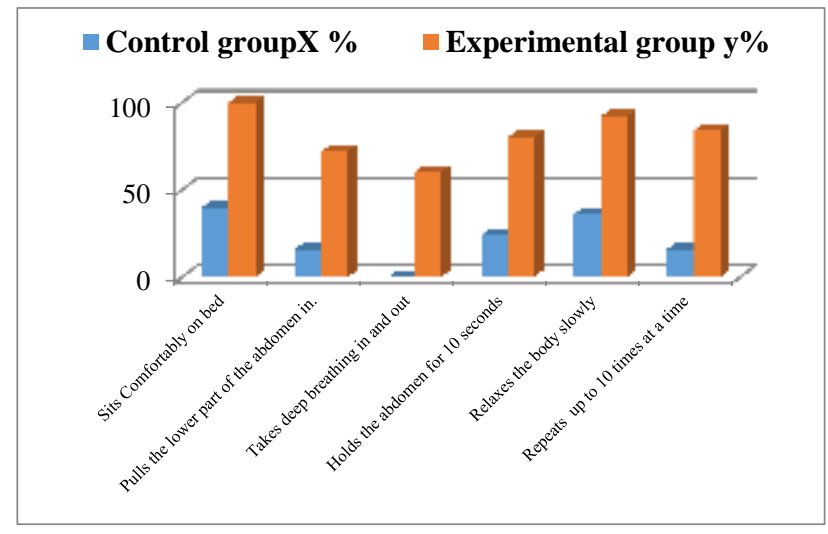

Figure 3: Items-wise comparison of control and experimental group correct practice scores regarding

Abdominal tightening exercise on observation.

Comparison of practice scores of control and experimental group regarding pelvic tilting shows that the highest percentage $36 \%$ of mothers had lie in half lying position. The higher $32 \%$ of mothers relaxed for sometimes. $28 \%$ of mothers breathed normally. More or less similarly $20 \%$ and $16 \%$ of mothers kept the feet flat on bed and bent her knee and placed one hand under curve of the abdomen. Similarly $15 \%$ of mothers and relaxed for sometimes. The lowest $12 \%$ of mothers hold breath up to 10 times and repeated all steps up to 10 times.

However in experimental group, the highest $92 \%$ mothers had lie in half lying position and breathed normally. The higher $84 \%$ of mothers placed one hand under curve the abdomen and relaxed sometimes. More or less similarly $80 \%$ and $76 \%$ of mothers tightens the abdomen and buttocks and bends her knees, kept the feet flat on the bed and, repeated up to 10 times. $72 \%$ of mothers hold breath up to $10 \mathrm{sec}$. The lowest $68 \%$ of mothers pressed the back down on underneath.

Further comparison of difference between control and experimental group, the highest $76 \%$ of mothers tightens the abdomen and buttocks and the lower $52 \%$ mothers relaxed for sometimes, reveals that the effectiveness of the Teaching Programme on pelvic tilting exercise (Figure 4).

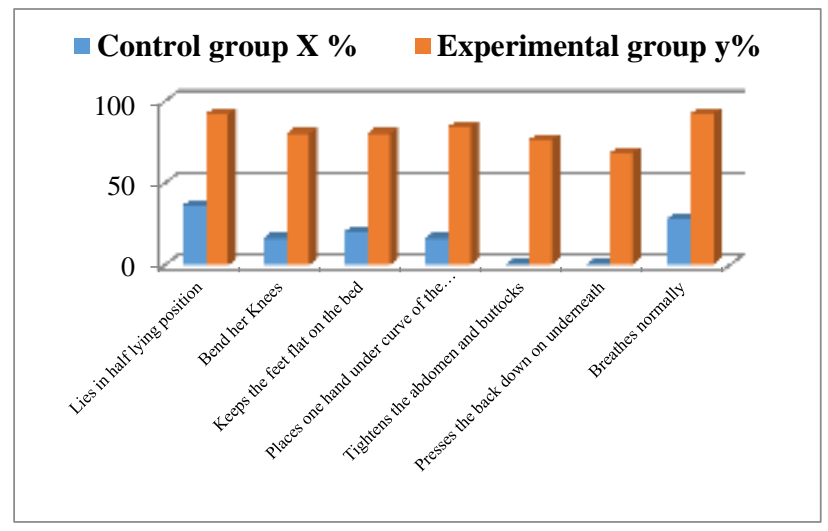

Figure 4: Items-wise comparison of control and experimental group correct practice scores regarding pelvic tilting exercise.

Comparison of practice scores of control and experimental group observation regarding knee rolling exercise shows that the highest $40 \%$ of mothers had lie on back lying position. The higher $32 \%$ of mothers relaxed for sometimes. Similarly $20 \%$ of mothers bend her knees and repeated 10 times for each side .similarly $16 \%$ of mothers pulled the abdomen in and rolled both knees on one side. $12 \%$ of mothers pulled in the lower part of the abdomen again and the lowest $08 \%$ mothers rolled both knees to the other side.

However in experimental group, $84 \%$ of mothers bent her knees. Similarly $80 \%$ mothers had lie in back lying position and relaxed for sometimes.76\% of mothers pulled the lower part of abdomen in and rolled the both knees on one side and rolled both knees to the other side. Similarly $72 \%$ of mothers pulled the lower part of abdomen in again and repeated 10 times all steps for each side. Similarly $68 \%$ of mothers kept the shoulder flat. The lowest $60 \%$ of mothers returned the knee to upright position. 
Further comparison of difference between control and experimental group, shows the highest $76 \%$ of mothers pulled the lower part of abdomen in and rolled the both knees on one side. The lowest $40 \%$ of mothers had laid in back lying position reveals that the effectiveness of the Teaching Programme on knee rolling exercises (Figure 5).

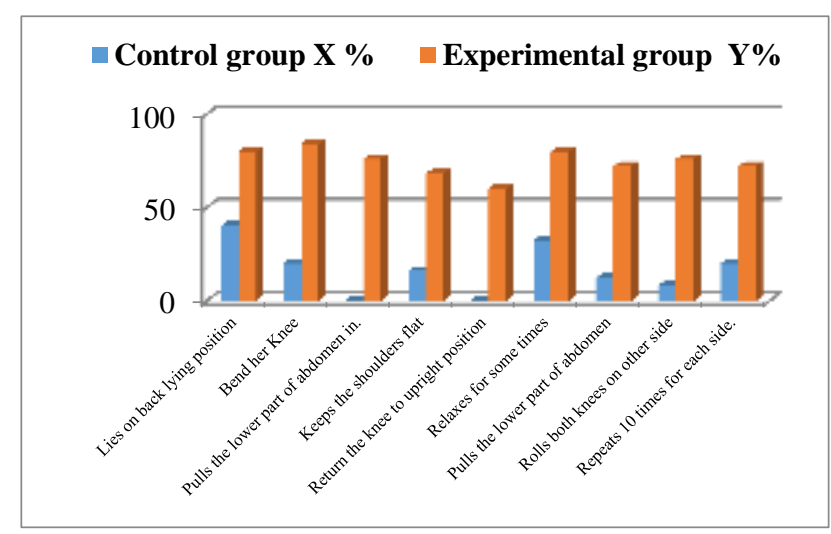

Figure 5: Items-wise comparison of control and experimental group correct practice scores of regarding Knee rolling exercise on observation.

\section{- Control group X\% Experimental group Y\%}

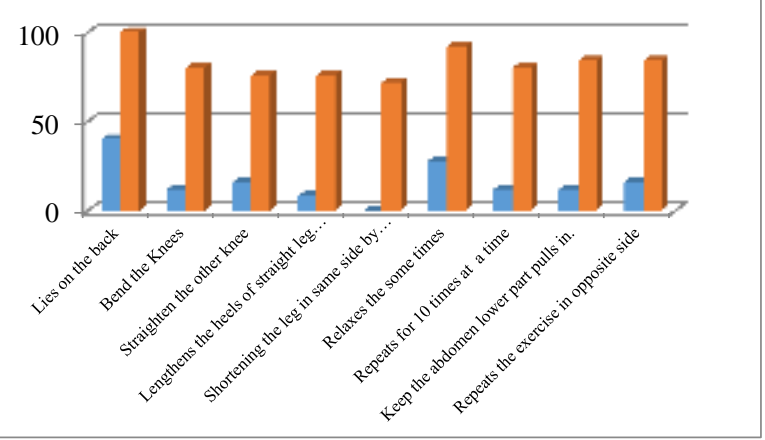

Figure 6: Items-wise comparison of control and experimental group correct practice regarding hip hitching exercise on observation.

Comparison of practice scores of control and experimental group regarding hip hitching exercise shows that the highest $40 \%$ of mothers from control group had lie on back. The higher $28 \%$ of mothers relaxed for sometimes. Similarly $16 \%$ of mothers straightened the knee and repeated the exercise in opposite side. $12 \%$ of mothers Bent the knee. Similarly $12 \%$ of mothers repeated all the steps for 10 times at a time and kept the abdomen lower part pulled in. The lowest 08\% lengthened the heels of straight leg downwards. However in experimental group, all of mothers $100 \%$ had lie on the back lying position. The highest $92 \%$ of mothers relaxed for sometimes. The higher $84 \%$ of mothers kept the abdomen lower part pulled in and repeated the exercise on opposite side. More or less similarly $80 \%$ of mothers repeated all steps 10 times at a time and bent the knees.
Similarly $76 \%$ of mothers straightened the knee and lengthened the heels of straight leg downwards. The lowest $72 \%$ of mothers shortened the leg in same side by drawing the hip up towards the rib. Further difference between control and experimental group, shows the highest $72 \%$ of mothers shortened the leg in same side by drawing the hip up towards the rib and kept the abdomen lower part pulled in. The lowest $60 \%$ of mothers had lie on the back lying position and straightened the knee reveals that the effectiveness of the Teaching Programme on hip hitching exercise (Figure 6).

\section{DISCUSSION}

Analysis result showed that the overall mean percentage for the experimental group overall $79.67 \%$. Area wise in the experimental group the highest mean percentage on hip hitching exercise were (7.44 \pm 2.64 ) which is $82.66 \%$ of the maximum scores. The lowest mean percentage on knee rolling $(6.68 \pm 0.95)$ which is $74.22 \%$ scores with a different of $8.44 \%$.Its seems that the teaching programme regarding postnatal exercises overall were effective. Analysis result showed that the overall mean percentage for the control group was $19.60 \%$. Area wise in control group the highest mean percentage on circulatory exercise were $(1.68 \pm 1.72)$ which is $28 \%$. The lowest mean percentage on hip hitching $(1.44 \pm 2.90)$ which is $16 \%$ of the maximum scores with a different of $12 \%$. Analysis result showed that the overall area-wise comparison between the mean score value of control and experimental group shows that, the highest mean percentages $(66.66 \%)$ for hip hitching exercise and the lowest $(50.66 \%)$ for abdominal breathing exercise. Areawise analysis of mean score show that control group the highest mean percentage score for circulatory exercise, which is $(28 \%)$ and lowest mean percentage (16\%) for hip hitching exercise, whereas for experimental group the highest mean percentage score for hip hitching exercise $(82.66 \%)$ and lowest mean percentage $(74.22 \%)$ for knee rolling with the effectiveness of $(60.07 \%)$ over all itemwise comparison of control and experimental group correct practice scores, The Planned Teaching Programme was highly effective regarding practice of place one hand on the chest and the abdomen in abdominal breathing exercise, tightens the abdomen and buttocks in Pelvic tilting exercise, and pulls the lower part of abdomen in knee rolling exercise. Moderate effectiveness was found regarding Hip hitching exercise, pelvic tilting and abdominal breathing exercises. Lowest effectiveness was found for the practice of rotated the feet at circle the feet at the ankle at least 12 times and hold tight both knees for a count of four in circulatory exercises, had lie on back lying position in knee rolling exercise, listened the breathing in abdominal breathing exercises. Significant difference was found between control and experimental group practice score $(\mathrm{t}=1.711)$ $(\mathrm{P}<0.05)$ Thus the calculated value is higher when compared to table value, so true hypothesis is accepted its indicating the effectiveness of planned teaching programme. 
Funding: Rajiv Gandhi University of Health Sciences, Karnataka, Bangalore, India

Conflict of interest: None declared

Ethical approval: The study was approved by the Institutional Ethics Committee

\section{REFERENCES}

1. Breslin ET. Women's Health Nursing E1-Sevior Science. Saunder's Company, Philadelphia; 2003:1897-1898.

2. Bryan A. Nursing practice in Midwives' Nightingale nursing. 2009;6(6):22.

3. Daftary SN, Chakravarthi S. Manual of Obstetrics. $1^{\text {st }}$ Edition, Elsevier India (p) Ltd. Reed Elsevier India Pvt. Ltd. 2011.

4. Ahmed N. A study of cesarean birth in a teaching Hospital. Indian Journal of medical and Research. 2006;1(3):75-9.

5. Benett RV. Myles Textbook for Midwives. $14^{\text {th }}$ edition, Churchill Livingstone London. 2006:583586.

6. Brunner. Suddarth's. Text Book of Medical Surgical Nursing. $10^{\text {th }}$ ed. Lippincott company Philadelphia, 452-453.

7. Burrows JL. Material morbidity associated with vaginal versus cesarean delivery. Obstetrics and Gynecology. 2008;10,103(5-1):907.

8. Gabbe GS. Obstetrics Normal and problem Pregnancies .4th edition, Churchill Livingstone, New York; 2007:562-567.

9. Ladewing F. Maternal New Born Nursing. 4th edition. Mosby Publications, Philadelphia; 2004:480488.

10. Polit DF, Hungers BP. Nursing. Research principles and methods. 6th ed. Philadelphia: Lippincott publishers. 1999:155.

11. Ahmed. A study of cesarean birth in teaching Hospital. Indian Journal of Medical. 2006;41(3):2919.

12. Bahague DP. To investigate why some women prefer cesarean section and how decisions medicals' birthings are influenced by patients. British Medical Journal. 2002;324(20):992-45.

13. Berghott T. Gynecological. Danish Obstetrics Personal Preference and General attitude to elective cesarean section on maternal requests. Alta Obstetrics. 2007;83(3):262-6.

14. Davis JD. Extended Preoperative hospitalization and prolonged bed Rest after operation lead to Deep Vein Thrombosis, American Journal of obstetrics and Gynecology. 2001;184(4):759-66.

15. Gynecology.2003, 40. Graham W.J. Introduction to Cesarean Birth Canadian Journal of Obstetrics and and 607-19., 173(112): P:.

16. Monica SR. Prophylactic use of antibiotics for nonlaboring Patients undergoing C.S. with intact membrane American Journal of Obstetrics and Gynecology. 2001;184(4):651-659.

17. Rojamma. M. teaching third Trimester Pregnant Women about New Born Care, the Indian Journal of Nursing and Midwifery, 2010;2(2):3656.

18. Ventolini. G. Decreasing infections morbidity in cesarean delivery by changing gloves. Journal of Reproductive medicine. 2004;49(1):13-6.

19. James DK. High Risk Pregnancy Management Options, 2nd edition. W. B. Saunders company, Philadelphia, 2007 and 1227., P: 1217 -.

20. Luckmann, Sorenson, Medical Surgical Nursing Post Operative Care W.B. Saunders company, Philadelphia, 2008:411-412.

21. Nagralh A. Progress in Obstetrics and Gynecology-I. Jaypee Brothers, New Delhi; 2003:694-699.

22. Noursis. Post Partum Care what's best for mother and babies Safe, Motherhood. 2008;2(24):4-8 .

23. Pellitteri A. Maternal and Child Health Nursing. $3^{\text {rd }}$ Edition. Philadelphia. Lippincott Company; 1999:527-545.

24. Watson's. Clinical Nursing and Related Science, $6^{\text {th }}$ ed. London: Baillie's Tindav (P) Ltd, 2002:187.

25. Publications, 25. Sharma J.B. The Obstetrics Protocol. Jaypee, New Delhi; 2005:164-166.

26. Hunt Leigh, Extended Preoperative Hospitalization and prolonged Bed rest after the operation lead for DVT, American Journal of Obstetrics and Gynecology. 2008;78(32).

Cite this article as: Rani VM, Andarieh MG,

Heidary FH. Effectiveness of planned teaching programme on practice of post natal exercises among mothers who have undergone lower segment cesarean section. Int J Reprod Contracept Obstet Gynecol 2016;5:3782-8. 